\title{
Case Report \\ Successful Removal of a Chronic Aspirated Foreign Body after Twelve Years
}

\author{
Radhika Z. Reddy $\mathbb{D}^{1},{ }^{1}$ Yvonne M. Carter, ${ }^{2}$ and David W. Hsia ${ }^{3}$ \\ ${ }^{1}$ VA Long Beach Medical Center, Department of Medicine, Division of Pulmonary and Critical Care Medicine, USA \\ ${ }^{2}$ Sinai Hospital of Baltimore, Department of Surgery, Division of Thoracic Surgery, USA \\ ${ }^{3}$ Harbor-UCLA Medical Center, Department of Medicine, Division of Respiratory and Critical Care Physiology and Medicine, USA \\ Correspondence should be addressed to Radhika Z. Reddy; rzopey@gmail.com
}

Received 28 February 2018; Accepted 17 April 2018; Published 31 May 2018

Academic Editor: Mark E. Wylam

Copyright (C) 2018 Radhika Z. Reddy et al. This is an open access article distributed under the Creative Commons Attribution License, which permits unrestricted use, distribution, and reproduction in any medium, provided the original work is properly cited.

Chronic retention of aspirated foreign bodies is rare but can result in indolent systemic and respiratory symptoms. Bronchoscopy may show features of tissue reaction to the foreign body, including granulation tissue, endobronchial stenosis, strictures, edema, and airway distortion. The diagnosis of foreign body aspiration is often difficult to establish since some patients may not give a clear history of aspiration or may present late. In addition, patients may be misdiagnosed with chronic pneumonia, bronchitis, asthma, or malignancy. We present the case of a 42-year-old male who had a chronically retained piece of an aluminum beverage container in the left mainstem bronchus for 12 years. Careful history, radiographic evaluation, and bronchoscopic examination revealed the foreign body, which was successfully extracted by rigid bronchoscopy.

\section{Case Presentation}

A 42-year-old man presented with a nine-year history of intermittent productive cough. He also endorsed recurrent episodes of fevers, chills, and night sweats but denied shortness of breath, chest pain, hemoptysis, or weight loss. He presented to the Emergency Department for similar symptoms two months prior and was given a seven-day course of levofloxacin, with temporary improvement in symptoms. Past medical history was significant only for diabetes mellitus. He had no prior surgery and did not take any medications. He worked as a gardener and denied any history of smoking, alcohol, or drug use. On examination, vital signs were stable with normal oxygen saturation on room air. Chest auscultation revealed mildly decreased breath sounds and rhonchi in the left lower lung field. The remainder of the examination was normal.

A chemistry panel and $\mathrm{CBC}$ were unremarkable, including a WBC of 7.0. Sputum AFB smears and bacterial cultures were all negative. Chest radiograph showed left basilar lung consolidation with tree-in-bud opacities and stenosis of the
LMSB (Figure 1(a)). Chest CT scan showed extensive treein-bud opacities with confluent consolidation in the left lung base, enlarged hilar and mediastinal lymph nodes, and stenosis of the LMSB (Figure 1(b)).

Flexible bronchoscopy revealed near-occlusion and distortion of the mid-LMSB (Figure 2(a)). Mechanical debulking with endoscopic biopsy forceps allowed for passage of the bronchoscope to the distal LMSB where a metallic foreign body was appreciated (Figure 2(b)). Histologic analysis of the biopsied endobronchial tissue revealed inflammation and squamous metaplasia, but no evidence of malignancy.

Subsequently, the patient underwent rigid bronchoscopy and a 2.7 centimeter metal foreign body was retrieved using rigid bronchoscopy forceps (Figure 2(c)). Granulation tissue occluding the LMSB was treated with argon plasma coagulation. The patient reported immediate improvement in his symptoms. Upon further questioning, he recalled a previous aspiration of a small piece of an aluminum beverage container that he used in lieu of dental floss 12 years before. This object was retained over that time period until we removed it. 


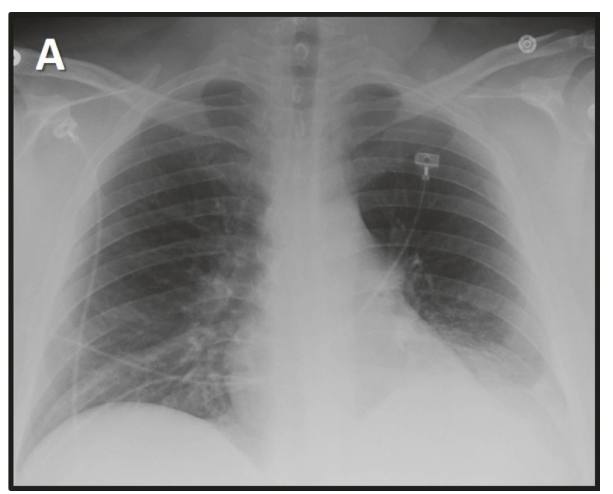

(a)

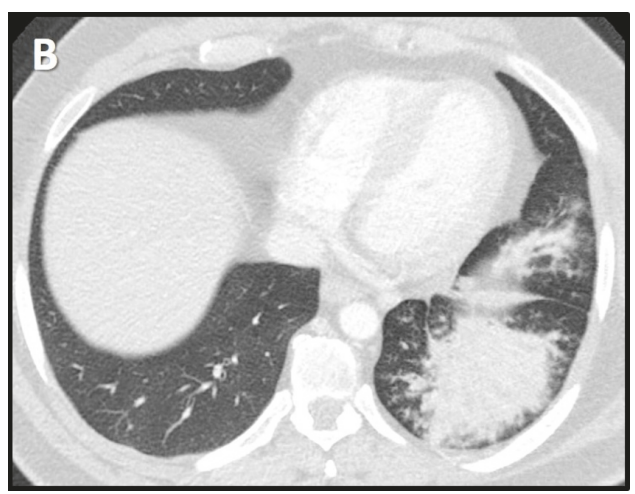

(b)

FIGURE 1: Chest radiograph (a) and computerized tomography (b) demonstrate left basilar lung opacification.

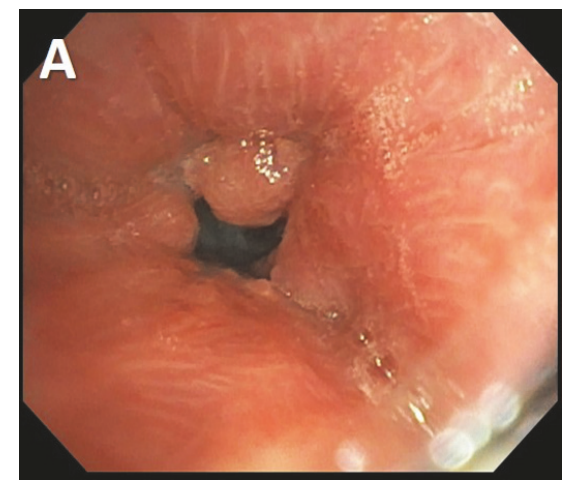

(a)

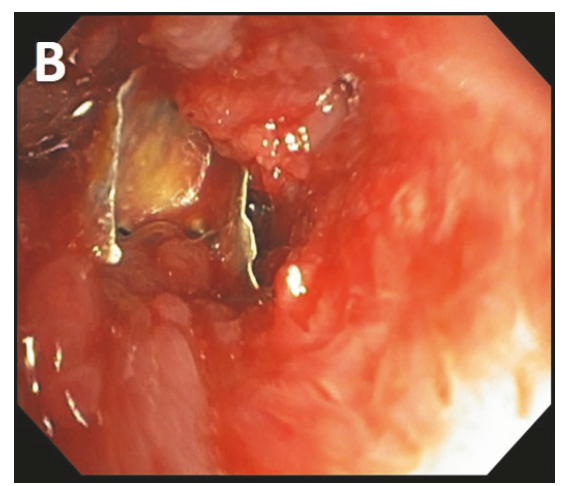

(b)

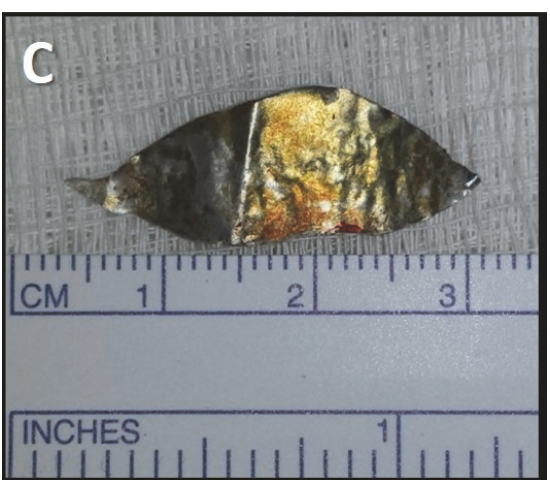

(c)

FIGURE 2: (a) Left mainstem bronchus with near-occlusion from obstructing granulation tissue. (b) Metallic foreign body appreciated in the distal left mainstem bronchus. (c) Metallic foreign body after removal.

Follow-up surveillance bronchoscopy demonstrated persistent endobronchial stricture in the distal LMSB. Rigid bronchoscopy with radial incisions by electrocautery knife and balloon dilation was used to restore luminal patency. Repeat CT scan one month after endobronchial therapy showed resolution of the left lung tree-in-bud opacities and consolidation and restored patency of the LMSB. The patient's chronic cough resolved and he remains asymptomatic.

\section{Discussion}

Foreign body aspiration is uncommon in healthy adults and occurs more commonly in children and in the elderly [1]. In adults, the incidence of foreign body aspiration peaks in the sixth decade of life. Adults who present with foreign body aspiration often have an underlying risk factor such as neuromuscular disease, altered mental status, head or facial trauma, endotracheal intubation, dental procedures, underlying pulmonary disease, or sedative or alcohol use [2]. In addition, adults with an impaired swallowing reflex due to underlying medical conditions are also at higher risk of foreign body aspiration [2]. Commonly aspirated foreign bodies include metallic objects (pins, screws, and nails), organic objects including food particles, plastic objects, and teeth [3].

Foreign body aspiration can present as a life-threatening emergency with acute airway obstruction and respiratory failure requiring urgent intervention; however, most patients present with indolent chronic symptoms [4]. Chronic cough is the most common symptom and is present in two-thirds of patients. Other commonly reported symptoms include hemoptysis, fever, and dyspnea [4]. Radiographic features of foreign body aspiration include nonresolving pneumonia, atelectasis, unilateral hyperinflation, and localized bronchiectasis [5]. If the foreign body is radiopaque, it can be directly visualized on chest X-ray. However, it may be obscured by chronic parenchymal changes [5].

Bronchoscopic evaluation of the airways may directly visualize the foreign body if it was recently aspirated [6]. However, in the case of chronic aspiration, bronchoscopy may show features of tissue reaction to the foreign body, including granulation tissue, endobronchial stenosis, strictures, edema, 
and airway distortion $[4,6]$. In one series of patients undergoing bronchoscopy for suspicion of foreign body aspiration, the foreign body was encountered in 49 of the 65 patients (75\%) [3]. Other bronchoscopic findings included granulation tissue, mucosal edema, and bronchial stenosis. The right lower lobe bronchus (30\%) and left mainstem bronchus (20\%) were the most common locations of foreign bodies [3].

The diagnosis of foreign body aspiration is often difficult to establish in patients for several reasons [7]. Some may not give a clear history of aspiration or may present late, up to months to years after the initial aspiration event [8]. In one study, only $25 \%$ of patients presented within 7 days of the aspiration event [3]. In some patients, the clinical symptoms may be subtle and remain undetected for years, especially if a small foreign body lodges in lobar or segmental bronchi [9]. One case report describes aspirated Coptis chinensis, a Chinese herbal medicine, for 10 years [10]. Another case series described a piece of metal in a patient's right lung for 27 years following an industrial accident [11], while the longest described foreign body retention is 40 years [2]. These cases of chronic retention of foreign bodies in the airways are very rare, and our patient is amongst the few described extending beyond a decade.

Occult foreign bodies may also be discovered incidentally when bronchoscopy is performed to evaluate a chronic cough, hemoptysis, or nonresolving pneumonia [12]. In addition, patients may be misdiagnosed with chronic pneumonia, bronchitis, asthma, or malignancy. Therefore, a recurrent pneumonia that fails to respond to antimicrobial therapy should prompt bronchoscopic examination for evaluation of obstruction by a foreign body or tumor [13].

Definitive treatment of foreign body aspiration requires removal of the foreign body. The first step in management of suspected foreign body aspiration in adults is a flexible bronchoscopy [14]. Extraction of the foreign body by flexible bronchoscopy is successful in approximately $90 \%$ of patients [3]. The advantages of initial flexible bronchoscopy include cost effectiveness and the ability to be performed as an outpatient [14]. Rigid bronchoscopy is pursued in cases where flexible bronchoscopy is unsuccessful or inadequate for safe extraction and simultaneous airway management [14]. In addition, if foreign bodies are impacted by significant granulation tissue or are difficult to grasp with flexible forceps due to size or shape, rigid bronchoscopy should be used for extraction. Endobronchial ablation, cryotherapy, or airway dilation techniques may be necessary in cases where foreign body retention has caused significant granulation tissue or airway stenosis [15]. Our case demonstrates that successful bronchoscopic removal may still be possible despite airway changes that occurred secondary to the aspirated foreign body over a 12year period.

\section{Abbreviations}

AFB: Acid fast bacilli

CBC: Complete blood count

CT: Computed tomography

CXR: Chest radiograph

LMSB: Left mainstem bronchus

WBC: White blood count.

\section{Conflicts of Interest}

The authors declare that there are no conflicts of interest regarding the publication of this paper.

\section{Disclosure}

This research did not receive any specific grant from funding agencies in the public, commercial, or not-for-profit sectors.

\section{References}

[1] F. Baharloo, F. Veyckemans, C. Francis, M. Biettlot, and D. O. Rodenstein, "Tracheobronchial foreign bodies: presentation and management in children and adults," CHEST, vol. 115, no. 5, pp. 1357-1362, 1999.

[2] A. H. Limper and U. B. S. Prakash, "Tracheobronchial foreign bodies in adults," Annals of Internal Medicine, vol. 112, no. 8, pp. 604-609, 1990.

[3] I. S. Sehgal, S. Dhooria, B. Ram et al., "Foreign body inhalation in the adult population: experience of 25,998 bronchoscopies and systematic review of the literature," Respiratory Care, vol. 60, no. 10, pp. 1438-1448, 2015.

[4] C.-H. Chen, C.-L. Lai, T.-T. Tsai, Y.-C. Lee, and R.-P. Perng, "Foreign body aspiration into the lower airway in Chinese adults," CHEST, vol. 112, no. 1, pp. 129-133, 1997.

[5] R. Zissin, M. Shapiro-Feinberg, J. Rozenman, S. Apter, J. Smorjik, and M. Hertz, "CT findings of the chest in adults with aspirated foreign bodies," European Radiology, vol. 11, no. 4, pp. 606-611, 2001.

[6] A. J. Rodrigues, E. Q. Oliveira, P. R. Scordamaglio, M. G. Gregório, M. Jacomelli, and V. R. Figueiredo, "Flexible bronchoscopy as the first-choice method of removing foreign bodies from the airways of adults," Jornal Brasileiro de Pneumologia, vol. 38, no. 3, pp. 315-320, 2012.

[7] M. B. Ramos, M. Botana-Rial, E. García-Fontán, A. FernándezVillar, and M. G. Torreira, "Update in the extraction of airway foreign bodies in adults," Journal of Thoracic Disease, vol. 8, no. 11, pp. 3452-3456, 2016.

[8] L. Lin, L. Lv, Y. Wang, X. Zha, F. Tang, and X. Liu, “The clinical features of foreign body aspiration into the lower airway in geriatric patients," Clinical Interventions in Aging, vol. 9, pp. 1613-1618, 2014.

[9] M. Blanco Ramos, A. Fernández-Villar, J. E. Rivo et al., "Extraction of airway foreign bodies in adults: experience from 19872008," Interactive CardioVascular and Thoracic Surgery, vol. 9, no. 3, pp. 402-405, 2009.

[10] L. Wang, B. Pudasaini, and X.-F. Wang, "Diagnose of occult bronchial foreign body: A rare case report of undetected Chinese medicine aspiration for 10 long years," Medicine (United States), vol. 95, no. 31, Article ID e4076, 2016.

[11] A. Debeljak, J. Šorli, E. Mušič, and P. Kecelj, "Bronchoscopic removal of foreign bodies in adults: experience with 62 patients from 1974-1998," European Respiratory Journal, vol. 14, no. 4, pp. 792-795, 1999.

[12] K. Mise, A. J. Savicevic, N. Pavlov, and S. Jankovic, "Removal of tracheobronchial foreign bodies in adults using flexible bronchoscopy: Experience 1995-2006," Surgical Endoscopy, vol. 23, no. 6, pp. 1360-1364, 2009.

[13] B. J. Alharthi, I. Masoodi, M. A. Almourgi, and S. Alzahrani, "Occult foreign body in the lung mimicking bronchogenic carcinoma," BMJ Case Reports, vol. 2014, Article ID 207438, 2014. 
[14] O. Dikensoy, C. Usalan, and A. Filiz, "Foreign body aspiration: clinical utility of flexible bronchoscopy," Postgraduate Medical Journal, vol. 78, no. 921, pp. 399-403, 2002.

[15] Y.-F. Fang, M.-H. Hsieh, F.-T. Chung et al., "Flexible bronchoscopy with multiple modalities for foreign body removal in adults," PLoS ONE, vol. 10, no. 3, Article ID e0118993, 2015. 


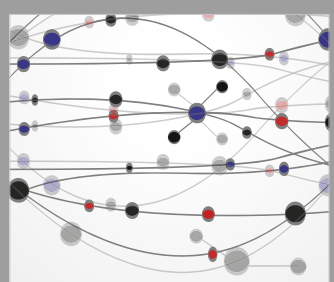

The Scientific World Journal
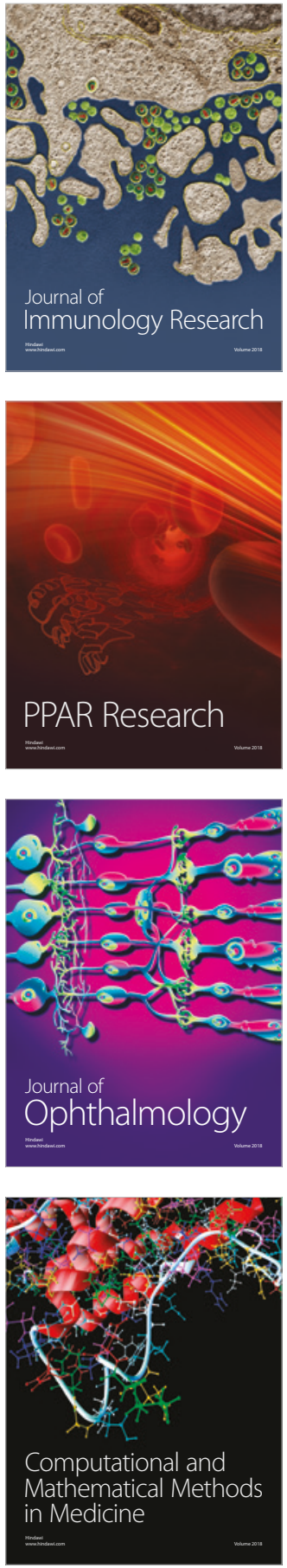

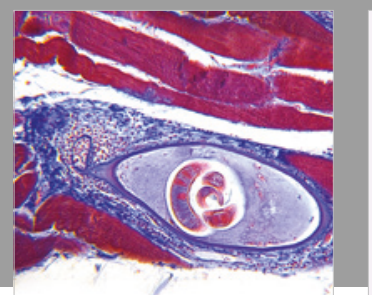

Gastroenterology Research and Practice

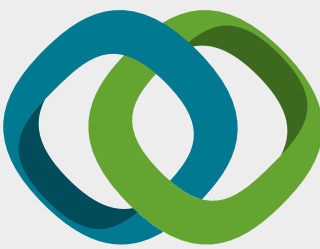

\section{Hindawi}

Submit your manuscripts at

www.hindawi.com
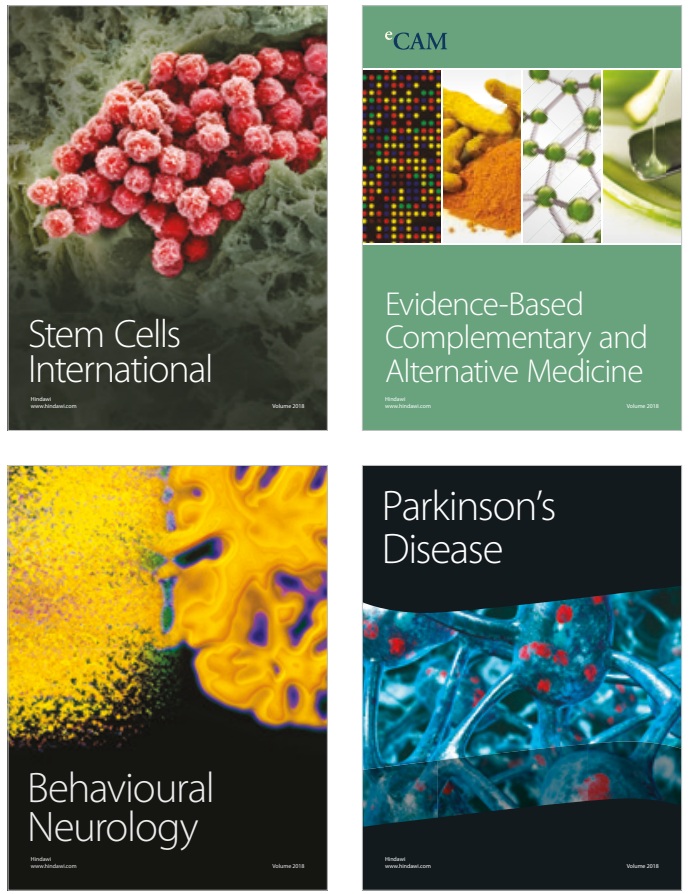

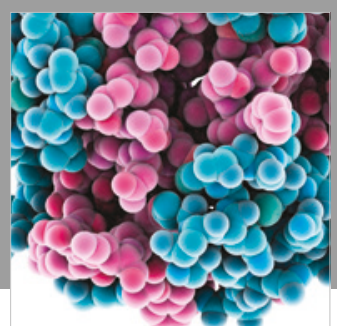

ournal of

Diabetes Research

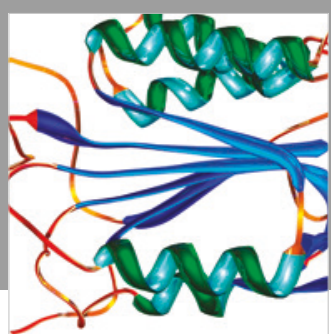

Disease Markers
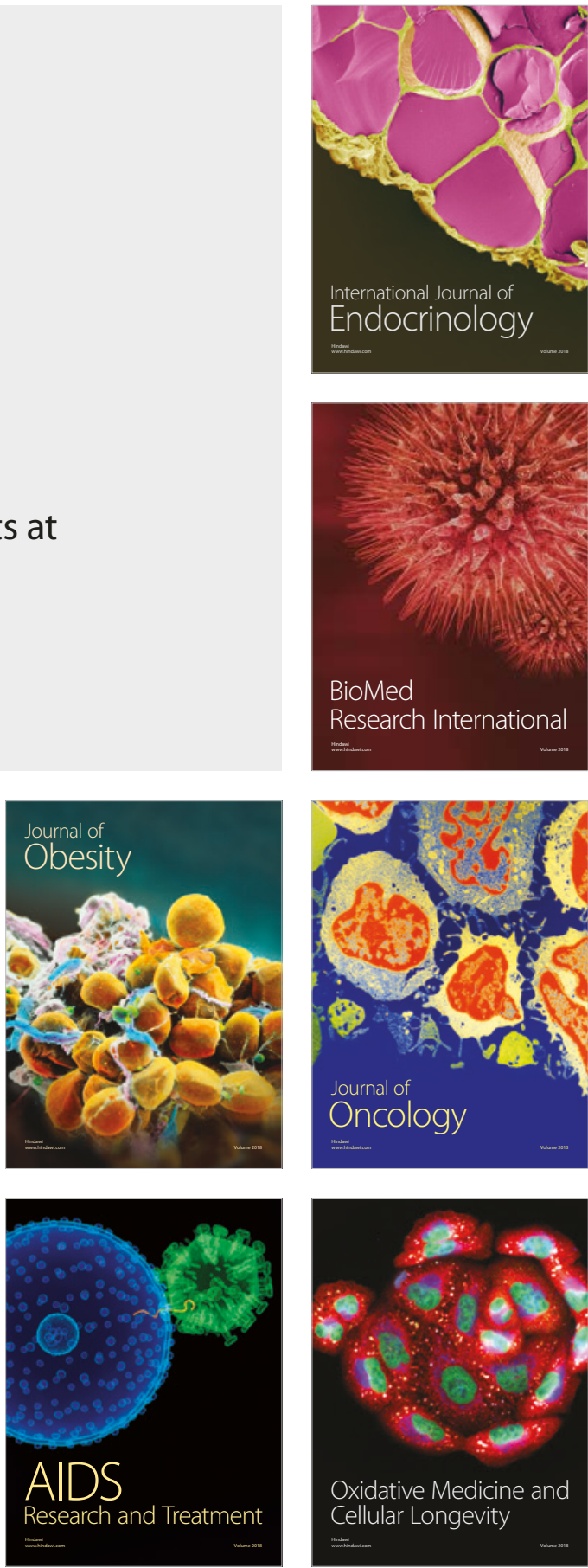